\title{
The Reasons and Countermeasures of Psychological Problems of Freshman
}

\section{from Rural Areas}

\author{
Yi Zou ${ }^{1, a}$, Zichao Wang ${ }^{2, b}$ \\ ${ }^{1,2}$ Sichuan University of Science \& Engineering, Zigong, Sichuan, 643000 \\ ${ }^{a}$ email, ${ }^{b}$ email
}

\section{Keywords: Freshman Rural Students; Problems; Causes; Countermeasures}

\begin{abstract}
The psychological problems of college students from a rural area are more common and special than those from the city. The period of the first year in university plays a key part for students to shift the role from a high school student to a university student. At this stage, rural students face a lot of psychological problems. Studies have shown that there are four main reasons leading to so many psychological problems of freshman from rural area: the big economic and cultural gap between urban and rural, the lower cultural level and less developed educational concept of the rural parents, the lack of psychological health education in basic education and the poor psychological quality of students. To solve these problems, we need to concentrateon above four aspects and take some effective measures. The society should implement its economic strategy to narrow the gap between rural and urban. Also, the system of mental health educationshould cover the whole society. Parents need to know more mental health education knowledge to understand and communicate with their children. Schoolsshould improve mental health education and increase the intensity of the funding for poor students from a rural area. Personally, rural students should improve their psychological quality and understand themselves in a comprehensive way.
\end{abstract}

\section{Introduction}

Nowadays, the mental health of college students has been highly concerned by the whole society. For educators, they should not only improve the overall level of mental health of college students, but also pay attention to the psychological problems of some students. Studies have indicated that the psychological problems of the students in rural areas are more serious than the students from the city.In general, the mental health status of students from rural areas is lower than urban students and poor college students mental health is serious than the non-poor students. ${ }^{1}$

The rural students mainly referto the students from the rural growth environment in rural areas whose parents are farmers or migrants. The first year is the key period for the students to enter the University from the high school. After entering the University, they face many problems of learning and great change of the living environment in the first year. There are some typical problems such as learning, life and interpersonal and psychological problems.

\footnotetext{
${ }^{1}$ Wang Zhixue: A comparative study on the mental health of college students in rural and urban Areas (D). Changchun: Northeast Normal University, 2007,11-12.
} 
In order to solve these problems, this paper focuses on four aspects: society, school, family and individual reasons. After exhaustive analysis, we put forward some related countermeasures to improve the level of mental health of the freshmanfrom the countryside and improve their all-round development.

\section{The Reasons for the Psychological Problems of the Freshman from Rural Area}

There is a huge difference between urban and rural areas caused by the two elements of the structure of urban and rural areas. On the one hand, the quality educational resource in rural areas is much less than that of the city such as the lack of excellent teachers, the lack of access to new information, serious shortage of educational facilities, etc.. On the other hand, there is a big gap between urban and rural culture. As a result, the behavior and values of urban and rural college students are very different. Moreover, due to the rapid transformation of Chinese society, social problems in rural areas have become increasingly prominent, such as the left behind children. These experiences have some effect on rural students in their growth stage. These students, after entering the university, in the process of contacting with the urban students, unable to adapt to these changes, cannot effectively regulate its emotion. Thus, some students produce some problem like anxiety, depression, low self-esteem, social attack, force, impulse and mental illness and psychological problems.

It's worth mentioning that though some freshman from rural with the will that do some part-time job to subsidize the cost of schooling or living fee, as a new student enrolling in the university, they have no special work skills, just can only do some simple jobs, such as leaflets, which lead to they feel upset, paying but no gaining. Therefore, economic pressure is also easy to lead to serious rural students with psychological problems.

The overall cultural level of rural parents is relatively low, so in the process of children' growth, a lot of parents to educate their children in a relatively simple way and they can't give their child the beneficial guidance, much less the knowledge of mental health education. They view their children' education as a task with only high scores and ignore the cultivation of students' comprehensive quality.

In today's society, with the rapid development of society and the change of social ideas, these parents without advanced knowledge of education will face several challenges in their children' educational process. Due to the lack of the latest educational concepts, educational methods, they cannot fully understand their children' ideas, cannot communicate with them well, and cannot properly guide their children. When children encounter difficulties, setbacks and psychological problems, it is difficult for parents to provide the right support. Even many rural parents stubbornly believe that their responsibility is just to provide the material conditions for the growth of the children, and teachers and school should take the responsibilities once their children entered the university.

In conclusion, above the rural parents' cultural level, the concept of education and other issues will directly influence to the rural students' mental health status after entering the university. Some students' backward ideas and experiences intensify mental health problems they may face.

In recent years, our schools at all levels have paid more and more attention to mental health education. The majority of schools have established a preliminary psychological health education work mechanism and equipped with full-time and part-time psychological health education work team. However, system construction and the work team construction still have many imperfections like all levels of the system cannot effectively play a synergistic effect, the number of personnel in quality is not up to the requirements. In university, education also base on the knowledge education, not combine the psychological health education and the comprehensive development of college students. Preach more, and practice less. The mental health class doesn't work and some students think that the course is not 
important at all. And the hardware and software of psychological consultation room cannot satisfy the needs of educators and education. The psychological counseling teachers also didn't major in psychology, which keeps impact on the effects of psychological counseling or other psychological health education.

In addition, due to the limited educational resources in rural areas and the not enough education, some learning problems appear after students entered the university. Before college life, their learning methods are relatively simple and their horizonis relatively narrow, while contact with new things, new technologies, new ideas in the University, their learning, life and many other aspects are prone to produce problems. For example, rural students, in the stage of basic education, lack of English and computer courses than urban students, after entering the University, the skill's gap between them will be bigger and bigger. Lacking of preparation and understanding about college study, lacking of plan and purpose to learn, students from rural have a tendency to produce some psychological problems.

Before entering the University, rural students' vision is not comparatively narrow. They just have one goal-enter the university gate. After entering the University, when facing the complex situation, they didn't prepare very well of psychological preparation, have no clear self-positioning and comprehensive self-cognition, so that their own life experience, habits of solving the problem, the world outlook, life outlook and values are not enough to support their successful integration into the university life. Thus, confused feeling and lose sense appeared.

With leaving the familiar rural environment, these students are confused by the problems of interpersonal communication. They have low self-esteem, self-closing, and do not take the initiative to say hello to others. They don't take participate in collective activities and have narrow communication space. Moreover, compared with urban students, rural students are not good at dealing with the opposite sex. Studies have shown that in high school, the city students' ratio of having romanced each other is usually higher than rural students. This gap is also easy to bring them to psychological problems.

Due to the limit of the living environment, most of the rural students lack the guidance of college life experience. Therefore, they didn't prepare for the University's before entering it. Once the reality of the university life is beyond their imaginations, without enough preparations, they are unable to overcome the difficulties they faced. Psychological problems tend to appear. Part of the rural student's lack of clear life planning and career planning, and they feel confused about the future. Some of them set unrealistic college life goals, not having strict and feasible action plan, self-control. As a result, they cannot achieve their goal and even waste their four-year college life. Thus, they lack the sense of achievement and a sense of success. In turn, they are influenced by a negative impact on their mental health.

Part of the rural students, after achieving the goals of enrolling in university, they have no other objectives of life. In their opinion, if they entered the university, they have achieved parents' idea of leaving rural and going to the city. Apparently, this cannot be a correct understanding of the value, and they ignore the efforts in the university. Seeing their classmates' colorful and successful college life, they have the feeling of flagging, anxiety, depression. At this time, some rural students lack of ability of integrating with their own resources, or the ability to mediate their own mental state, so that there are some psychological problems.

Also, the stigmas of mental health problems make the spreading of mental health knowledge sluggish, as well as affected the rural students cognitive with mental health problems. Thus, they lost their own internal support when psychological problems appeared.

\section{To Solve the Psychological Problems of Freshman from Rural Area}

The psychological problems faced by the many freshmen from rural area are both universal and specific, 
which need not only to carry out the general psychological health education, but also solve their psychological problems in a targeted way. To solve the psychological problems of freshman from rural areas is a systematic project, which needs the social, school, family and personal collaborate with each other and solve it step by step.

A large part of the psychological problems of the freshman from rural areas related to the two element structure of urban and rural areas. However, social reform will not happen overnight, and the construction of a comprehensive harmonious society has a long way to go. Fundamentally, to improve the level of mental health of the students in the countryside, on the one hand, more resources should be devoted to the construction of the new countryside, especially to the construction of rural basic education, which can promote equality of urban and rural education. On the other hand, it's necessary to build a better city with excellent culture, which can truly achieve the chant of "Better City, better life". 2

Also, the government and all sectors of society can establish and improve the mental health service system, increase input on mental health of society, allocate medical resources reasonably, andestablish a wide range of national mental health care system. Meanwhile, we must strengthen the propaganda of 'mental health law' and other laws and regulations to let people know more about legal issues related to psychological issues and improve the level of mental health law. Strengthen the publicity of mental health knowledg, so that people can improve understanding of mental health problems, eliminate the misunderstanding of cognition, and all the people are devoted to mental health work.

Family status is directly related to a person's mental health level, and even is the most critical factor in a period of a person's growth. However, due to the influence of education level, society culture and traditional concept, rural parents don't understand the child's psychological health education, or do not pay enough attention to it. Lacking of training the children' comprehensive quality and the consciousness or idea of the overall development of the child, the child's overall health development is not guaranteed, even more, their mental health growth is influenced by these disadvantages.

Therefore, parents should learn more about the relevant knowledge of education, to understand the rules of the growth ofthe child, to obey the child's growth principles. They should be concerned about the healthy growth and development of their children. After their kids go to college, they should care more about their psychological health status, timely understand their situation and difficulties, and give them more support, encouragement and help more than just the cost of living. It's irresponsible for parents to take their children to school and care about nothing.

Due to the rapid changes in the society and the generation gap, naturally, there are more and more communicative problems between rural students and their parents. Still, the main measure to guarantee the rural mental health of college students is keeping the family warm and harmonious and concern about the child's psychological status. Unfortunately, some children already have psychological problems, so parents and school teachers should work together to seek professional help, to jointly cope with the relevant issues.

Schools should focus on strengthening the supervision of education, improve the management ability, and pay attention to students' mental health education from the beginning of the university. Also, university should strengthen psychological health education in terms of publicity and make a full use of a mental health survey. It's necessary to pay attention to these students and timely solve the problem. At the same time, strengthen the system construction, the establishment of three systems of psychological health education center: university psychological health, psychological counseling center, and

\footnotetext{
${ }^{2}$ The theme of 2010 Shanghai EXPO
} 
psychological health associations or grassroots educational center of mental health. These methods can enhance the management level of the school and education in a wide range and in a higher level.

About the daily propaganda, it's critical to form a psychological atmosphere whichcannotonly be beneficial to the positive psychology, but also in time to find the problem. In the two mental health day of '3.25' (Be best to yourself) and '5.25' (Love yourself), in all kinds of activities and means of publicity, school can preach the positive publicity concept of mental health. In addition, about teachers, schools should recruit high-quality mental health education teachers and workers, strengthen the training of teachers, especially the counselor team, to enhance the level of teachers' psychological health education. Meanwhile, school should improve the hardware level of the mental health education such as the university's psychological consultation room, and promote mental health education from generalization to individuation.

It's imperative for schools' mental health work to care about freshman. Firstly, it is necessary to improve the funding system for poor students starting from their enrollment, which can let them feel warm, so that these students can bear unnecessary psychological pressure because of economic reasons. Secondly, for students who have difficulty in daily life, school should take some measure to help enhance their self-confidence, to help them solve the difficulties encountered in life, learning. Last but not least, to strengthen the communication and cooperation with professional psychological institutions is also a great way, which can help these students with the strength of professionals and institutions.

If psychological problems happen to a freshman from rural area, it is certain that it'll be difficult to overcome the problems by their own.

Firstly, they should be positive to find the cause and to strengthen self-cultivation by enhancing the humanities accomplishment. For example, they can read some books to comprehend its life, and watch the classic movie to enjoy the spirit of it. At this period, these freshmen should explore its advantages, be kind to themselves and the world from a positive point of view. At their daily life, they should record thankful thing of themselves and people around, accept their imperfections and failures, improve individual's psychological adjustment ability, improve their own personality, improve their self-control. And, instead of looking for external satisfaction, it is more significant to find the inner self-transcendence.

Secondly, on the basis of full understanding of themselves, they can make a clear career planning and life planning. It can be divided into several parts such as first semester planning, the first academic year planning, university life planning, career planning and life planning. Of course, with the new recognizes about life, college students can constantly revise the relevant planning according to the actual situation. After planning, they can make a strict schedule and implement it.

Furthermore, they can also make new friends to motivate themselves, contact with pretty good students to encourage each other.

\section{Conclusion}

The psychological health level of the freshmen from rural area is lower than that of the urban students.In the first year, students are prone to have psychological problems. Rural students' psychological problems are created from many ways including the economic gap between urban and rural areas, cultural differences, poor rural parents' education way, not enough cares from family, poor education of psychological health in school, insufficient individual ability of adjusting and so on. To solve the problem, from the point of view of social way, on the one hand, the wide gap between urban and rural areas should be narrow. On the other hand, mental health education system should cover the whole society. About family, in order to understand and communicate with their children. Parents need to know more mental 
health education knowledge. About school, they should strengthen the effectiveness of mental health education and increase the intensity of the funding for poor students in the countryside. Personally, rural students should improve their psychological quality and understand themselves in a comprehensive way. As a result, freshmen from rural areas can experience a colorful year and lay a foundation for their future life. Resourceful youths lead to a resourceful nation; strong youths lead to a strong country. Everyone in the country should engage in the work of youth.

\section{Reference}

[1] Wang Qun. College students' Mental Health Education (M). Shanghai: Fudan University press. 2005

[2] Yang Di. Study on psychological health of college students in rural areas of China (D). Hefei: HeFei University of Technology. 2007

[3] Zhao Xianwei. Problems and solutions of college students in rural poverty -- Taking Yantai University as an example (D). Beijing: Chinese Academy of Agricultural Sciences. 2009

[4] Sun Xueqin. Study on mental health status and Countermeasures of rural college students (D). Shaanxi: Northwest Agriculture and Forestry University, 2013

[5] Zhao Xia Xue, Lu Li, Liang Zhi Qun. Rural College Students Mental Health Survey (J). Chinese Journal of public health, 2008 August Vol 24 No. 8, 928-929.

[6] Xia Xia. Perspective and resolution of psychological problems of rural students (J). Research on education and teaching, April 2009, 17-25. 